\title{
Controversias actuales en el diagnóstico de la diabetes mellitus gestacional
}

\author{
José Fernando Botero Arango ${ }^{1,5}$; Claudia Monsalve Arango ${ }^{2}$; Alex Ramírez Rincón ${ }^{2}$; Natalia \\ Aristizábal Henao ${ }^{2}$; José Luis Torres Grajales ${ }^{3,5}$; Esdras Martín Vásquez Mejía ${ }^{1}$; Alberto Villegas \\ Perrase $^{4}$; Andrés Felipe Palacio Barrientos ${ }^{1,5}$
}

\begin{abstract}
${ }^{1}$ Especialista en Medicina Interna y Endocrinología Clínica. Clínica Integral de Diabetes - CLID. Medellín, Colombia.

${ }^{2}$ Especialista en Medicina Interna. Residente de Endocrinología, diabetes y metabolismo del adulto, Universidad Pontificia

Bolivariana. Medellín, Colombia.

${ }^{3}$ Especialista en Medicina Interna y Endocrinología Clínica. Clínica Las Américas. Medellín, Colombia

${ }^{4}$ Especialista en Medicina Interna y Diabetología. Clínica Integral de Diabetes - CLID. Medellín, Colombia.

${ }^{5}$ Profesor programa Endocrinología, diabetes y metabolismo del adulto. Universidad Pontificia Bolivariana. Medellín, Colombia.
\end{abstract}

Dirección de correspondencia: José Fernando Botero Arango. Correo electrónico: josebotero1971@gmail.com

Declaración de conflictos de interés: Los autores declaran no presentar ningún conflicto de interés.

\section{Resumen}

$\mathrm{L}$ a diabetes gestacional es una condición que se reconoce por primera vez en el embarazo y está asociada a serias complicaciones maternas, fetales y perinatales en el corto y largo plazo. El tema más controversial ha sido la forma para realizar el diagnóstico por la existencia de múltiples criterios diagnósticos. Esta situación se ha vuelto más compleja en los últimos años a partir de la implementación de la estrategia de un paso, propuesta por la International Association of Diabetes and Pregnancy Study Groups (IADPSG, por sus iniciales en inglés), cuya aplicación aumentó la incidencia de la enfermedad hasta un $17,8 \%$ en comparación con la estrategia tradicional de dos pasos (tamización y prueba confirmatoria), con la que la incidencia siempre se había estimado entre 6 y 7\%. La evidencia actual no ha demostrado que el enfoque de un paso sea superior a la estrategia de dos pasos, en términos de complicaciones, costos y salud pública. Dados los beneficios de la intervención, por disminución de la incidencia de desenlaces adversos como macrosomía, distocia de hombros, recién nacidos grandes para la edad gestacional e hipertensión materna, se justifica la realización del tamizaje universal en las gestantes.

Palabras clave: Diabetes gestacional, diagnóstico, embarazo, prueba de tamizaje.

\section{Abstract}

Gestational diabetes is any glucose abnormality first recognized in pregnancy. It has been associated with maternal, fetal and newborn complications. Which diagnostic method to use has been a source of controversy (75 gram OGTT vs the two step approach 50 gram followed by 100 gram OGTT). Since the recommendations made by the International Association of Diabetes and Pregnancy Study Groups (IADPSG) of using the 75 gram OGTT it has been argued that the incidence would rise from approximately $6-7 \%$ to $17,8 \%$. There is no evidence that the one step strategy is superior to the two step approach in terms of reduction of complications or from an economical stand point. Regardless of the method used to make the diagnosis it has been shown that treatment of GDM (gestational diabetes) decreases the incidence of large for gestational age, shoulder distocia, macrosomia and maternal hypertension. Universal screening should be recommended to all pregnant patients.

Key words: Gestational diabetes, diagnosis, pregnancy, screening

\section{Introducción}

La diabetes mellitus gestacional (DMG) se define como cualquier grado de intolerancia a la glucosa que inicia o se reconoce por primera vez durante el embarazo ${ }^{(1)}$. Esta definición tiene limitaciones, ya que no tiene en cuenta el tratamiento utilizado, no reconoce si la enfermedad persiste después del embarazo y no diferencia a las pacientes que tienen diabetes o intolerancia a los carbohidratos antes de la gestación ${ }^{(1-3)}$. El término propuesto por la Endocrine Society es "hiperglucemia en el embarazo", el cual es apropiado y contemporáneo con base en la evidencia disponible ${ }^{(4)}$.

La DMG se asocia con complicaciones maternas, como hipertensión gestacional, preeclampsia, parto pretérmino y aumento de riesgo de parto por cesárea. Las complicaciones perinatales incluyen macrosomía, hipoglucemia neonatal, hiperbilirrubinemia, hipocalcemia, policitemia, nacimiento por cesárea, síndrome de dificultad respiratoria, distocia de hombros y trauma al 
nacer ${ }^{(5,6)}$. Después del parto, las pacientes tienen un alto riesgo de volver a desarrollar diabetes gestacional, prediabetes o $\mathrm{DM}^{(7)}$. Si bien en los trabajo, originales de 0'Sullivan se estimó que el riesgo de desarrollar diabetes tipo 2 en las mujeres con antecedentes de DMG era de 50\% en el seguimiento 22 a 28 años después del embarazo ${ }^{(8)}$, estudios en mujeres latinoamericanas han demostrado que hasta $60 \%$ de las diabéticas gestacionales pueden evolucionar a DM tipo 2 a los cinco años ${ }^{(8,9)}$.

\section{Métodos}

Se presenta una revisión narrativa de la literatura sobre el diagnóstico de la DMG. Como estrategia de búsqueda se utilizó la base de datos Medline desde enero de 1995 hasta enero de 2015 con los siguientes descriptores "gestational diabetes mellitus", "diagnosis", "epidemiology" y "screening". Como criterios de selección se incluyeron artículos en inglés y español del tipo ensayo clínico, consensos, revisiones narrativas, metanálisis y revisiones sistemáticas. De la lista de artículos identificados por esta estrategia de búsqueda y teniendo en cuenta el gran número de publicaciones sobre el tema y la restricción del espacio, se seleccionaron las publicaciones más relevantes sobre el diagnóstico de la DMG. Se adicionaron dos referencias en junio de 2015 por actualización de guías nacionales e internacionales.

\section{Epidemiología}

La prevalencia de la DMG puede variar según la incidencia de diabetes tipo 2 en la población estudiada ${ }^{(5)}$, la edad y el peso de la materna ${ }^{(10)}$. La estrategia de tamizaje utilizada, también puede determinar la prevalencia de la enfermedad. Cuando se utilizan los criterios clásicos (prueba de tolerancia oral a la glucosa con 50 gramos seguida de una segunda prueba con 100 gramos de glucosa), se ha estimado que la prevalencia de la DMG se sitúa entre el 6 y el 7\%. Si se utilizan los criterios diagnósticos derivados del estudio HAPO (Hyperglycemia and Adverse Pregnancy Outcome $)^{(11)}$ que fueron recomendados en el 2010 por la International Association of Diabetes and Pregnancy Study Groups (IADPSG), la incidencia puede aumentar hasta $17,8 \%$ al realizar una única prueba de tolerancia oral a la glucosa con 75 gramos $^{(12)}$.

En Colombia se ha reportado una prevalencia menor a la informada en la literatura. En una publicación de la ciudad de Medellín del año 2000, la prevalencia encontrada fue de 1,43\% aplicando los criterios diagnósticos del National Diabetes Data Group (NDDG, por sus iniciales en inglés) y con los criterios de Carpenter y Coustan aumentó a 2,03\%(13,14).

\section{Diagnóstico de DMG}

En la actualidad, el diagnóstico de la DMG se realiza entre las semanas 24 y 28, utilizando alguna de las siguientes estrategias (tabla 1):

- Estrategia de un paso: Prueba de tolerancia oral a la glucosa con 75 gramos (PTOG-75 g).

- Estrategia de dos pasos: Tamizaje con carga de $50 \mathrm{~g}$ de glucosa, seguida de confirmación con una prueba de tolerancia oral a la glucosa con 100 gramos (PTOG-100 g).

Desde la descripción de la enfermedad por O’Sullivan y Mahan en la década de los sesenta, el diagnóstico de la DMG ha sido controversial porque los criterios iniciales fueron diseñados para identificar el riesgo de desarrollar diabetes después del embarazo, sin tener en cuenta el riesgo de eventos adversos perinatales $^{(12)}$. Hasta el año 2010, la estrategia diagnóstica de dos pasos había sido utilizada de forma sistemática en la práctica obstétrica, donde el debate estuvo dirigido a la aplicación de los criterios de Carpenter y Coustan, versus los de NDDG, y la discusión de tamización universal frente a la selectiva ${ }^{(3,12,15)}$.

En el año 2008 fue publicado el estudio HAPO que encontró una correlación directa entre el aumento de los valores de glucemia materna y los resultados adversos maternos y fetales, independiente de otros factores de riesgo, por lo que la IADPSG publicó en el año 2010 las nuevas recomendaciones para el diagnóstico y clasificación de la hiperglucemia en el embarazo, teniendo como punto central la estrategia de un paso con PTOG-75 g, estableciendo como umbral arbitrario para el diagnóstico las cifras de glucemia a partir de las cuales

Tabla 1. Estrategias y umbrales para el diagnóstico de DMG

\begin{tabular}{|c|c|c|c|c|c|}
\hline Enfoque & Criterio & Ayunas mg/dl & 1 hora $\mathrm{mg} / \mathrm{dl}$ & 2 horas mg/dl & 3 horas $\mathrm{mg} / \mathrm{dl}$ \\
\hline \multirow{3}{*}{$\begin{array}{c}2 \text { pasos } \\
\text { PTOG-50 g } \\
\rightarrow \\
\text { PTOG-100 g }\end{array}$} & Carpenter y Coustan & 95 & 180 & 155 & 140 \\
\hline & NDDG & 105 & 190 & 165 & 145 \\
\hline & CDA (2013) & 95 & 191 & 162 & \\
\hline \multirow{3}{*}{$\begin{array}{c}1 \text { paso } \\
\text { PTOG-75 g }\end{array}$} & OMS & 126 & & 140 & \\
\hline & NICE & 100 & & 140 & \\
\hline & IADPSG & 92 & 180 & 153 & \\
\hline
\end{tabular}

Adaptado de $(18,23)$ 
la morbilidad es 1,75 veces la media de la población en relación a tres variables: peso al nacer, adiposidad abdominal y niveles de péptido $C$ en sangre del cordón ${ }^{(11,12,16,17)}$. La aplicación de estos criterios ha aumentado la controversia por el aumento de la prevalencia de la DMG hasta 15 a $20 \%$, lo cual triplica en la práctica el número de pacientes con DMG, causando un impacto significativo en los costos de la atención médica, la capacidad de los servicios de salud y una medicalización de embarazos que anteriormente serían considerados como normales $^{(1,12,18)}$

A raíz de esta situación, los Institutos Nacionales de Salud de los Estados Unidos (NIH, por sus iniciales en inglés) establecen su posición, en marzo de 2013, en el Consensus Development Conference on Diagnosing Gestational Diabetes Mellitus, recomendando continuar con la estrategia de dos pasos para el diagnóstico de DMG, ya que los criterios IADPSG aumentan la prevalencia de diabetes gestacional con consecuencias importantes desde el punto de vista de la salud pública. Se presentaría un aumento de la tasa de cesáreas, valoración más intensiva del neonato, aumento de los costos directos e indirectos, aumento de las cargas psicosociales y la interrupción en la vida normal de las pacientes ${ }^{(18)}$. Los NIH concluyen que no hay evidencia suficiente que demuestre que el enfoque diagnóstico de un solo paso genere beneficios en la madre o en el recién nacido pero sí es cierto que aumenta las intervenciones y los costos de la atención en salud ${ }^{(18)}$. Posteriormente, el American College of Obstetricians and Gynecologists (ACOG), de forma oficial, acogió los argumentos de los NIH, recomendando utilizar la estrategia de dos pasos para el diagnóstico de $\mathrm{DMG}^{(5)}$.

Los beneficios de la detección de la DMG a través de la estrategia de dos pasos están soportados en dos grandes estudios de intervención que compararon el tratamiento consistente en dieta, automonitoreo e insulina, en caso de ser necesario, versus el cuidado usual prenatal en enfermedad leve en los que $80-90 \%$ de las pacientes se controlaron solo con $\operatorname{dieta}^{(16,19-22)}$.

En el Australian Carbohydrate Intolerance Study in Pregnant Women (ACHOIS), en el que se incluyeron mil pacientes, se encontró que la tasa de complicaciones perinatales medidas por el desenlace compuesto de: muerte, distocia de hombros, fracturas óseas y parálisis del plexo braquial fueron menores en el grupo de intervención con manejo nutricional individualizado, automonitoreo cuatro veces al día y terapia con insulina en caso de no lograr control glucémico (1 vs. 4\%, RR 0,33 IC95\% 0,14-0,75), al igual que la frecuencia de recién nacidos con peso $>4.000 \mathrm{~g}$ y la incidencia de preeclampsia. Se observó una mayor tasa de admisión a cuidado intensivo neonatal y una mayor tasa de inducción del parto en el grupo de intervención con tasas similares de cesárea y disminución de los índices de depresión a los tres meses posparto ${ }^{(5,16,20)}$.

El estudio de Landon et al. para el Eunice Kennedy Shriver National Institute of Child Health and Human Development
Maternal-Fetal Medicine Units Network (MFMU) incluyó 958 embarazadas, de las cuales 485 se aleatorizaron al grupo de intervención mediante asesoramiento nutricional, dieta e insulina en caso de ser necesario y 473 al grupo control, encontrando que no había reducción en el desenlace compuesto de mortalidad perinatal, trauma al nacer, hipoglucemia, hiperbilirrubinemia o hiperinsulinemia neonatal (32,4 vs. 37\%) pero que en los desenlaces secundarios se observó una menor frecuencia de recién nacidos con peso $>4.000 \mathrm{~g}$, de la tasa de cesáreas, menos distocia de hombros y menos trastornos hipertensivos en el grupo de intervención ${ }^{(5,16,21)}$.

En el 2011, la American Diabetes Association (ADA) recomendó la realización de la PTOG-75 g para el diagnóstico de DMG, pero desde los Standards of Medical Care in Diabetes del año 2014 permite utilizar la estrategia de un paso (consenso IADPSG) o la de dos pasos (consenso NIH), porque no hay datos suficientes para demostrar la superioridad de una estrategia $^{(1,2,22)}$

La Endocrine Society, en las guías de diabetes y embarazo del año 2013, recomienda la tamización universal para diabetes de forma temprana en la gestación (antes de la semana 13 o tan pronto como sea posible) con glucosa plasmática en ayunas, HbA1c o glucosa aleatoria, utilizando los criterios convencionales para el diagnóstico de DM (tabla 2). En la semana 24-28 de gestación, la Endocrine Society recomienda la realización de estudios para DMG en las mujeres sin diagnóstico previo con la estrategia de un paso, utilizando la PTOG-75 g con los criterios IADPSG (tabla 3) ${ }^{(4)}$.

Tabla 2. Criterios de DM en el primer trimestre según la Endocrine Society

\begin{tabular}{l|c|c|c}
\hline \multicolumn{1}{c|}{ PTOG-75 g } & $\begin{array}{c}\text { Glucosa en } \\
\text { ayunas } \mathbf{~ m} \text { /dl }\end{array}$ & $\begin{array}{c}\text { Glucosa } \\
\text { aleatoria } \mathbf{m g} / \mathbf{d l}\end{array}$ & HbA1c\% \\
$\begin{array}{l}\text { Diabetes mellitus } \\
\text { pregestacional }\end{array}$ & $\geq 126$ & $\geq 200$ & $\geq 6,5$ \\
\hline $\begin{array}{l}\text { Diabetes } \\
\text { gestacional }\end{array}$ & $92-125$ & No aplica & No aplica \\
\hline
\end{tabular}

Tomado de (4)

Tabla 3. Criterio de DMG entre las semanas 24 y 28, según la Endocrine Society

\begin{tabular}{l|c|c|c}
\multicolumn{1}{c|}{ PTOG-75 g } & Ayunas $\mathbf{~ g / d l ~}$ & $\mathbf{1}$ hora $\mathbf{~ m g / d l}$ & $\mathbf{2}$ horas $\mathbf{~ g / d l}$ \\
$\begin{array}{l}\text { Diabetes mellitus } \\
\text { pregestacional }\end{array}$ & $\geq 126$ & No aplica & $\geq 200$ \\
\hline $\begin{array}{l}\text { Diabetes } \\
\text { gestacional }\end{array}$ & $92-125$ & $\geq 180$ & $153-199$ \\
\hline
\end{tabular}

Tomado de (4) 
Como variación a la estrategia de dos pasos, la Canadian Diabetes Association (CDA) tiene como primera elección el tamizaje con PTOG-50 g con realización de un segundo paso si la glucemia es mayor de $140 \mathrm{mg} / \mathrm{dl}$ con una PTOG-75 g con medición en ayunas y cada hora poscarga por 2 horas haciendo el diagnóstico de DMG con uno o más valores alterados (tabla 1). Esta estrategia de enfoque secuencial utilizada en Canadá desde hace varios años tiene la posibilidad de realizar el diagnóstico de DMG solo con la alteración del primer paso si la PTOG-50 g es > 200 mg/dl. Desde el año 2013, el comité de expertos de la CDA ha preferido utilizar en el segundo paso con la PTOG-75 g los umbrales correspondientes a un OR 2,0 de los desenlaces primarios del estudio HAPO, lo cual ha generado un cambio mínimo a la guía previa del año 2008. Como estrategia diagnóstica alterna, la Canadian Diabetes Association sugiere la aplicación del enfoque de un paso con los criterios IADPSG ${ }^{(18,19)}$.

La Organización Mundial de la Salud (OMS) también ha propuesto estrategias de un paso para el diagnóstico de diabetes gestacional con medición de la glucemia en ayunas y a las dos horas, siendo diagnóstico un solo valor alterado, pero su uso no ha sido muy extendido por aumentar el número de diabéticas gestacionales $\sin$ un beneficio adicional( ${ }^{(18)}$. Las guías NICE (National Institute for Health and Clinical Excellence) formuladas en el año 2008 en el Reino Unido, con base en análisis económicos en salud, habían recomendado utilizar la estrategia de un paso con la PTOG-75 g, utilizando los criterios de la OMS; sin embargo, la actualización publicada en el año 2015 ha modificado los umbrales diagnósticos para DMG, siendo diagnósticos si hay valores mayores de $100 \mathrm{mg} / \mathrm{dl}$ en ayunas o de $140 \mathrm{mg} / \mathrm{dl}$ a las dos horas poscarga (tabla 1) ${ }^{(23)}$.

Para el año 2015, el Ministerio de Salud de Colombia, a través de la Alianza CINETS para el desarrollo de Guías de Práctica Clínica (GPC), ha formulado la "Guía de práctica clínica para el diagnóstico, tratamiento y seguimiento de la diabetes gestacional", que hace una recomendación fuerte a favor de la estrategia de un paso, aplicando los criterios IADPSG a todas las embarazadas, a partir de la semana 24 , para la realización del diagnóstico de la DMG (tabla 1) ${ }^{(24)}$.

\section{Importancia del diagnóstico de DMG}

Un metanálisis y una revisión sistemática publicada recientemente sobre los beneficios y riesgos del tratamiento de la DMG incluyó cinco estudios aleatorizados, controlados y seis estudios de cohorte, encontrando evidencia moderada en favor del tratamiento para disminuir los desenlaces de preeclampsia (RR 0,62 [IC95\% 0,43 - 0,89]), distocia de hombros (RR 0,42 [IC95\% 0,22 - 0,77]) y macrosomía (RR 0,50 [IC95\% $0,35-0,71])$. No se encontró diferencia en la tasa de cesáreas, la inducción del parto, neonatos pequeños para la edad gestacional, admisión a unidad de cuidados intensivos neonatales, hipoglucemia neonatal o de alteraciones metabólicas a largo plazo. El aumento en la demanda de los servicios de salud fue el factor negativo más notable ${ }^{(25)}$.

Los estudios de intervención demuestran que la detección y el tratamiento de la DMG logran una reducción en la incidencia de macrosomía, distocia de hombros y el número de recién nacidos grandes para la edad gestacional, además de menor incidencia de hipertensión materna ${ }^{(16)}$. En este contexto, la identificación de las mujeres con diabetes en el embarazo tiene importancia clínica, ya que al ser una enfermedad asintomática se pueden disminuir los resultados perinatales adversos al hacer algún tipo de intervención ${ }^{(19)}$.

\section{Tamizaje con PTOG-50 g}

O'Sullivan y Mahan describieron el tamizaje de DMG con PTOG de 50 g en 1973, y en la actualidad es el método más común de tamización con umbral diagnóstico entre 130 y 140 $\mathrm{mg} / \mathrm{dl}$ sin encontrarse ensayos aleatorizados que determinen la superioridad de un punto de corte sobre otro. Diferentes estudios han demostrado que con un umbral de $140 \mathrm{mg} / \mathrm{dl}$ hay menor tasa de falsos positivos y un mejor valor predictivo positivo y que al disminuir el umbral a 130 o $135 \mathrm{mg} / \mathrm{dl}$ hay un aumento de la sensibilidad ${ }^{(5,26)}$.

En 2012, se publicó una revisión sistemática que evaluó la aplicabilidad de la PTOG-50 g como primer paso en el tamizaje de DMG seguido de la PTOG-100 g o PTOG-75 g que a un umbral de $140 \mathrm{mg} / \mathrm{dl}$ mostró una sensibilidad de 74\% (IC95\% 0,62-0,87) y una especificidad de 77\% (IC95\% 0,66-0,89) ${ }^{(27)}$. En el año 2013, el U.S. Preventive Services Task Force publicó otra revisión sistemática con resultados similares, encontrando que a un umbral de $140 \mathrm{mg} / \mathrm{dl}$ la sensibilidad es del 70 al 88\% y la especificidad del 69 al 89\% y que a un umbral de $130 \mathrm{mg} / \mathrm{dl}$ la sensibilidad es del 88 al 99\% y la especificidad del 66 al 77\%; esta publicación también evaluó la utilidad de la glucemia en ayunas a un umbral de $85 \mathrm{mg} / \mathrm{dl}$ con una sensibilidad del $87 \%$ y una especificidad del $52 \%{ }^{(25)}$. Ambas revisiones coinciden en la utilidad de la PTOG-50 g para identificar a las pacientes con $\mathrm{DMG}^{(25,27)}$.

En cuanto al umbral elegido, se espera que requieran PTOG$100 \mathrm{~g}$ entre el $14 \%$ y el $23 \%$ de las pacientes tamizadas. Es decir que, al utilizar como punto de corte $130 \mathrm{mg} / \mathrm{dl}, 23 \%$ de las pacientes requerirán una segunda prueba, y al aumentar el umbral a $140 \mathrm{mg} / \mathrm{dl}$ se hará la PTOG-100 g en $14 \%$ de las pacientes ${ }^{(18)}$.

En relación con la influencia de la etnicidad en la prueba de tamizaje, Esakoff et al. revisaron en 2005 una cohorte retrospectiva de 14.565 embarazadas que incluyó 1.789 latinas para determinar si había modificaciones en el diagnóstico de DMG con base en la raza, encontrando que había diferencias en la sensibilidad y especificidad de la PTOG-50 g entre los grupos étnicos examinados, siendo mayor en las pacientes afroamericanas. Este estudio propone una nueva aproximación al tamizaje de la DMG basado en el análisis de la sensibi- 
lidad, especificidad, puntos de corte superiores y la curva ROC para lograr una sensibilidad del $90 \%$ con una tasa de falsos positivos del $10 \%$, por lo que el umbral óptimo de detección sería de $135 \mathrm{mg} / \mathrm{dl}$ para las afroamericanas, $140 \mathrm{mg} / \mathrm{dl}$ para las razas blancas y latinas y $145 \mathrm{mg} / \mathrm{dl}$ para las asiáticas. Si se desea una sensibilidad mínima del 95\%, los umbrales óptimos serían $135 \mathrm{mg} / \mathrm{dl}$ para las afroamericanas y $130 \mathrm{mg} / \mathrm{dl}$ para todos los demás grupos étnicos ${ }^{(28)}$.

\section{Tamizaje universal versus selectivo}

En la Fourth International Workshop Conference on Gestational Diabetes Mellitus de 1997 se recomendó la tamización selectiva con base en factores de riesgo para mujeres con mayor probabilidad de padecer diabetes en el embarazo y en la vida tardía ${ }^{(3)}$. En la Fifth International Workshop Conference del año 2005 se mantuvo la misma postura y se discutió el beneficio y la costo-efectividad de la tamización en los subgrupos de bajo riesgo para DMG, los cuales representan un porcentaje menor al $10 \%$ de la población. El bajo porcentaje de pacientes excluidas hace poco justificable el enfoque de la tamización selectiva porque vuelve más complejo el proceso de detección de la diabetes gestacional (tabla 4) ${ }^{(3,15)}$.

Tabla 4. Estrategias de tamizaje para DMG según el riesgo en la Fifth International Workshop Conference on Gestational Diabetes Mellitus

\begin{tabular}{l} 
RIESGO DE DMG \\
Valoración del riesgo en el primer control prenatal \\
Bajo riesgo: La realización de estudios diagnósticos no es \\
rutinaria si se cumplen todos los criterios \\
\hline - $\quad$ Miembro de grupo étnico con baja prevalencia de DMG \\
- $\quad$ Edad menor de 25 años \\
- Peso normal antes del embarazo \\
- $\quad$ Sin historia personal de metabolismo anormal de los carbohi- \\
- $\quad$ dratos
\end{tabular}

Riesgo promedio: Realización de estudios diagnósticos entre las semanas 24 y 28 de gestación

- Estrategia de dos pasos

- Estrategia de un paso

\begin{tabular}{|c|}
\hline $\begin{array}{c}\text { Alto riesgo: Realización de estudios diagnósticos, tan pronto } \\
\text { como sea posible, si uno o más de los siguientes ítems están } \\
\text { presentes }\end{array}$ \\
\hline $\begin{array}{l}\text { - } \quad \text { Obesidad severa } \\
\text { - Historia familiar de DM tipo } 2 \\
\text { glucosa o glucosuria }\end{array}$ \\
\hline $\begin{array}{l}\text { Si no se realiza el diagnóstico de } \mathrm{DMG} \text {, se debe repetir el estudio } \\
\text { diagnóstico entre las semanas } 24 \text { y } 28 \text { de gestación o si la paciente } \\
\text { presenta signos o síntomas de hiperglucemia. }\end{array}$ \\
\hline
\end{tabular}

Tomado de las referencias $(3,15)$.
Tabla 5. Factores de riesgo que indican el estudio de diabetes en individuos asintomáticos

\section{Todos los adultos con sobrepeso (IMC $\geq 25 \mathrm{~kg} / \mathrm{m}^{2}$ ) y que tengan} factores de riesgo adicionales:

- Inactividad física

- Familiares en primer grado con diabetes

- Raza o etnia de alto riesgo (afroamericanos, latinos, nativos americanos, americanos asiáticos, nativos de las islas del Pacífico)

- Mujeres con hijos de más de 9 libras al nacer o que hayan tenido diagnóstico de DMG

- Hipertensión arterial (>140/90 mm/Hg o terapia para hipertensión)

- Colesterol $\mathrm{HDL}<35 \mathrm{mg} / \mathrm{dl}$ y/o triglicéridos $>250 \mathrm{mg} / \mathrm{dl}$

- Mujeres con síndrome de ovario poliquístico

- HbA1c > 5,7\%, alteración de la glucosa en ayunas o PTOG medidos previamente

- Otras condiciones asociadas con resistencia a la insulina (obesidad severa, acantosis nigricans)

- Historia de enfermedad cardiovascular

Adaptado de la referencia (2)

Dada la epidemia de obesidad y diabetes tipo 2 en mujeres en edad reproductiva, en los Standards of Medical Care in Diabetes de la ADA se consideran otros factores de riesgo adicionales (tabla 5) a los ya mencionados en la International Workshop Conference, para el estudio de diabetes en pacientes asintomáticas, que deben ser considerados en la visita prenatal inicial que hacen razonable realizar tamizaje para DM tipo 2, usando los criterios estándar de la Endocrine Society ${ }^{(2,4,15)}$.

Aunque el estudio Toronto Trihospital Gestational Diabetes Project que evaluó estrategias de tamización selectiva para diabetes gestacional mostró una reducción de casi $35 \%$ en las pruebas diagnósticas realizadas con similar tasa de detección a las del cuidado estándar ${ }^{(29)}$, se ha considerado que el tamizaje selectivo puede no identificar hasta un tercio de las pacientes con DMG. Varios estudios han evidenciado claramente que la ausencia de factores de riesgo para DMG no se asocia con mejores resultados maternos y fetales ${ }^{(30,31)}$.

Un estudio irlandés del año 2000 comparó las estrategias de tamización selectiva versus tamización universal, utilizando la PTOG-50 g para tamización, seguida de una prueba con PTOG-100 g. Se encontró que la estrategia universal detecta una prevalencia de DMG de 2,7\% mayor que el grupo de tamización selectiva (prevalencia de 1,45\%); esto permitió realizar un diagnóstico más temprano con menor tasa de cesáreas, prematuridad, preeclampsia y admisiones a cuidado intensivo neonatal ${ }^{(30)}$. McCarthy et al. evaluaron en Argentina la aplicación del tamizaje universal versus selectivo en 1.702 pacientes 
no seleccionadas con PTOG-7 5 g (diagnóstico a las dos horas $>140 \mathrm{mg} / \mathrm{dl}$ ), encontrando una prevalencia de DMG de 5,8\%. Los principales factores de riesgo en esta población fueron: la obesidad pregestacional, la hiperglucemia previa, la edad mayor de 30 años y la DMG previa. La prevalencia de DMG fue de $8,6 \%$ en las multigestantes con factores de riesgo para la enfermedad y alrededor de $4 \%$ en las multíparas sin factores de riesgo y en las primigestantes con y sin factores de riesgo, siendo esta última una cifra significativa que apoya la tamización selectiva ${ }^{(32)}$.

Un estudio retrospectivo francés de publicación reciente con 18.775 mujeres, que evaluó el valor diagnóstico y pronóstico de la tamización selectiva, encontró que 65,3\% de las pacientes con DMG $(\mathrm{N}=2.710)$ tenían al menos un factor de riesgo. Esto disminuiría los diagnósticos de DMG hasta en $25 \%$ de las pacientes de la cohorte al aplicar la estrategia selectiva. Aunque se determinó que había una correlación entre el número de factores de riesgo con la alta incidencia de eventos relacionados con DMG (preeclampsia, grandes para la edad gestacional y distocia de hombros); el desenlace de eventos relacionados con la enfermedad también fue significativo en las mujeres con DMG sin factores de riesgo, en comparación con las embarazadas no diabéticas, por lo que los autores no apoyan la tamización selectiva que actualmente se utiliza en Francia $^{(31)}$.

Recientemente, la cohorte irlandesa del ATLANTIC-DIP de Avalos et al., publicada en 2013 con 5.500 mujeres a las que se les realizó tamización universal con los criterios IADPSG entre 2007-2009, hizo un análisis de la aplicación de la tamización selectiva según factores de riesgo con base en las guías ADA, las guías NICE y las guías irlandesas, hallando que al aplicar las estrategias respectivas el 54, 58 y $76 \%$ de las pacientes tenía al menos un factor de riesgo. Sin embargo, uno de los resultados más importantes de este estudio fue evidenciar que podían ser diabéticas gestacionales sin factores de riesgo el $5 \%$ de las pacientes tamizadas selectivamente según la ADA, el 16\% según las guías irlandesas, y hasta el 20\% según las guías NICE, con el agravante de que los resultados maternos (tasa de cesáreas, desórdenes hipertensivos y polihidramnios) y perinatales (malformaciones congénitas y admisión a cuidado intensivo neonatal) fueron más ominosos en este grupo, en comparación con las pacientes no diabéticas. Las únicas características clínicas que permitieron clasificar a las pacientes en la categoría de bajo riesgo fueron la edad menor de 21 años y el IMC $<25 \mathrm{~kg} / \mathrm{m}^{2}$, pero solo $2 \%$ de la población estudiada tenía esas características. Con estos argumentos, la colaboración ATLANTIC-DIP ha recomendado el tamizaje universal para DMG, pero en caso de considerar la tamización selectiva se deben utilizar las guías de la ADA, ya que es la estrategia con menor probabilidad de omisión de diagnóstico ${ }^{(33)}$.

Similares resultados al ATLANTIC-DIP se demostraron en un estudio observacional retrospectivo en Australia que tam- bién validó las guías NICE, ADA y ADIPS (Australasian Diabetes in Pregnancy Society), al encontrar una alta sensibilidad del 92,7, 100 y 98,6\% y una baja especificidad del 32,4, 3,9 y $13,7 \%$ respectivamente. En esta publicación se concluyó que no hay ventajas significativas del tamizaje selectivo sobre el universal $^{(34)}$.

\section{Costo-efectividad de las estrategias diagnósticas}

Ante el aumento de la prevalencia de la DMG con los criterios IADPSG y las actuales limitaciones basadas en la evidencia de esta propuesta, es posible que la decisión sobre qué estrategia diagnóstica utilizar sea determinada por el impacto sobre la economía y la infraestructura de los sistemas de salud. Hasta ahora la mayoría de los análisis de costos apoyan la estrategia de dos pasos para el diagnóstico de $\mathrm{DMG}^{(19)}$.

En el sistema canadiense se realizó una evaluación de las tecnologías en salud. Las decisiones se basaron en un estudio prospectivo, controlado, aleatorio, diseñado para comparar tres métodos de detección de la DMG en el que se realizó un análisis de minimización de costos con 1.594 pacientes. Se evaluaron los costos médicos directos e indirectos, los costos no médicos directos y la prevalencia de la enfermedad. En el grupo 1 se usó el método de dos pasos con PTOG-50 g, seguido de PTOG-100 g con criterios NDDG; en el grupo 2 se usó el método de dos pasos con PTOG-50 g con posterior PTOG-75 g de acuerdo con los criterios CDA; y el grupo 3 utilizó el método de un paso con la PTOG-75 g utilizando los criterios CDA. El enfoque de dos pasos demostró ser menos costoso con igual poder diagnóstico, ya que la incidencia de DMG fue similar en los tres grupos $(3,7,3,7$ y $3,6 \%$ en los grupos 1,2 y 3 , respectivamente). Los costos totales por paciente tamizada fueron $\$ 91,61$ dólares canadienses en el grupo $1, \$ 89,03$ en el grupo 2 y $\$ 108,38$ en el grupo 3. Cabe aclarar que con el método de dos pasos el 39\% de las pacientes del grupo 1 y el $61 \%$ de las pacientes del grupo 2 no requirieron segunda prueba porque en las recomendaciones de la CDA, un tamizaje mayor de 186 $\mathrm{mg} / \mathrm{dl}$ es diagnóstico de $\mathrm{DMG}^{(19,35)}$.

En la actualidad comienzan a conocerse importantes estudios de análisis de impacto económico de las guías IADPSG. Se realizó un estudio en una cohorte teórica de 100.000 mujeres, comparando en un modelo analítico de decisiones el tamizaje con PTOG-75 g con criterios IADPSG versus el tamizaje con PTOG-50 g, encontrando que la estrategia de un paso es costoefectiva definida por la comparación de los costos totales con los QALYs (quality-adjusted life years) materno y fetales. Esto mejoraría los resultados maternos y neonatales al lograr el diagnóstico y tratamiento para DMG en $2 \%$ de pacientes adicionales $^{(36)}$.

Recientemente, Durán et al. publicaron el St. Carlos Gestational Diabetes Study, un estudio que comparó una cohorte de 
1.750 mujeres gestantes entre abril de 2011 y marzo de 2012, evaluadas mediante la estrategia de dos pasos (criterios de Carpenter y Coustan) entre las semanas 24 y 28 de gestación con una segunda cohorte de 1.526 gestantes evaluadas con la estrategia de un paso, usando los criterios IADPSG entre abril de 2012 y marzo de 2013; ambas cohortes recibieron la misma intervención y seguimiento para DMG. Se encontró que con el uso de los criterios IADPSG había un aumento en la tasa de DMG en comparación con la estrategia de dos pasos (35,5 vs. 10,6\%); sin embargo, se demostró mejoría en los resultados obstétricos y perinatales con el uso de los criterios IADPSG dada por disminución de la tasa de hipertensión gestacional, prematuridad, cesáreas, pequeños para la edad gestacional, grandes para la edad gestacional e ingreso a la unidad de cuidado intensivo neonatal. El análisis de costos demostró que, a pesar del aumento de la incidencia, el uso de los criterios IADPSG generó un ahorro estimado de 14.358,06 euros por cada 100 mujeres evaluadas por disminuir las cesáreas y por menos ingresos a la unidad de cuidado intensivo neonatal, en comparación con la estrategia de dos $\operatorname{pasos}^{(37)}$.

\section{Tamización de diabetes mellitus tipo 2}

En la cohorte original de 0’Sullivan se estimó que la prevalencia de DM tipo 2 era hasta de $60 \%$ en el seguimiento a 28 años después de la DMG.

Un estudio de cohorte retrospectiva realizado en Israel que incluyó 185.416 embarazadas, de las cuales el 6,07\% eran diabéticas gestacionales diagnosticadas por la PTOG-50 g o la PTOG-100 g demostró en el seguimiento posparto que el riesgo acumulado de diabetes a los 10 años en las pacientes con DMG fue de $15,7 \%$, en comparación con el 1\% de las pacientes no diabéticas gestacionales. Los principales factores de riesgo para la progresión fueron el número de valores anormales en la PTOG y el uso de insulina ${ }^{(38)}$. Un importante metanálisis del año 2009 que incluyó 20 estudios con 675.455 mujeres y 10.859 eventos de DM tipo 2, demostró que las mujeres con DMG tienen un aumento del riesgo de desarrollar DM 2, en comparación con las mujeres normoglucémicas en el embarazo (RR 7,43, IC95\% 4,79-11,51) ${ }^{(39)}$.

La recomendación actual de la ACOG para la búsqueda de DM 2 es la realización de glucemia en ayunas o PTOG-75 g entre las 6 y 12 semanas posparto. Ambas pruebas son adecuadas para el diagnóstico de diabetes, aunque la glucosa en ayunas tiene una menor sensibilidad para detectar otras formas de intolerancia a los carbohidratos ${ }^{(5)}$. Por esta razón, el Fifth International Workshop on Gestational Diabetes Mellitus y el Standards of Medical Care in Diabetes de la ADA recomienda únicamente realizar la PTOG-75 g aplicando los criterios es- tándar para el diagnóstico de diabetes ${ }^{(1,2,5,15)}$. La hemoglobina glicosilada (HbA1c) no se considera adecuada para el diagnóstico posparto de diabetes porque puede estar influenciada por el tratamiento anteparto de la hiperglucemia ${ }^{(2)}$.

Las pacientes que se diagnostiquen con diabetes deben remitirse para terapia farmacológica y terapia médica nutricional. En quienes se realice el diagnóstico de prediabetes por alteración de la glucosa en ayunas o en la prueba de tolerancia a la glucosa, las medidas iniciales tendrán como objetivo disminuir la incidencia de diabetes con la modificación en el estilo de vida con disminución de peso, aumento de la actividad física, terapia médica nutricional y metformina en casos seleccionados. En las pacientes con tamizaje posparto para diabetes dentro de límites normales, la ADA recomienda repetir los estudios cada tres años y sugerir cambios en el estilo de vida ${ }^{(5,8)}$.

\section{Conclusión}

La DMG es una condición que aumenta de forma significativa el riesgo de eventos adversos maternos y neonatales. Las pacientes con DMG tienen un mayor riesgo de desarrollar diabetes tipo 2. Esto justifica el tamizaje universal, el diagnóstico y el tratamiento de la DMG con beneficios demostrados por varios estudios de intervención ${ }^{(7)}$. La mayor controversia en DMG ha sido el proceso diagnóstico que ahora centra el debate en la selección de la estrategia de un paso, propuesta por la IADPSG que basa su recomendación en el estudio HAPO que no es un estudio de intervención versus la estrategia de dos pasos propuesta por el NIH y el ACOG, las cuales se basan en estudios de intervención como el ACHOIS y el MFMU. Hasta el momento, la falta de evidencia de la superioridad de una estrategia sobre la otra ha orientado la discusión pero probablemente sean los estudios recientes de costo-efectividad los que definan una tendencia. Se requieren ensayos clínicos de intervención y no solo observacionales en pacientes diagnosticadas por la estrategia de un paso que evalúen el impacto real del tratamiento de la diabetes durante el embarazo en términos de complicaciones maternas y perinatales. De acuerdo con las GPC desarrolladas por el Ministerio de Salud y la Alianza CINETS, Colombia ha adoptado la tamización universal y la estrategia diagnóstica de un paso con los criterios IADPSG. Desde el punto de vista de los autores de este artículo se considera que no se puede llegar a una conclusión tan contundente mientras no se tenga una evidencia que demuestre los beneficios reales que justifiquen aumentar la incidencia de la enfermedad al cambiar el criterio diagnóstico. Mientras no haya unanimidad, se seguirá observando una gran discrepancia en el modo de diagnosticar la DMG entre las grandes asociaciones científicas mundiales. 


\section{Referencias}

1. American Diabetes Association. Diagnosis and classification of diabetes mellitus. Diabetes Care. 2014;37 Suppl 1:S81-90.

2. American Diabetes Association. Standards of medical care in diabetes--2014. Diabetes Care. 2014;37 Suppl 1:S14-80.

3. Metzger BE, Coustan DR. Summary and recommendations of the Fourth International Workshop-Conference on Gestational Diabetes Mellitus. The Organizing Committee. Diabetes Care. 1998;21 Suppl 2:B161-7.

4. Blumer I, Hadar E, Hadden DR, Jovanovič L, Mestman JH, Murad MH, et al. Diabetes and pregnancy: an endocrine society clinical practice guideline. J Clin Endocrinol Metab. 2013;98(11):4227-49.

5. Committee on Practice Bulletins--Obstetrics. Practice Bulletin No. 137: Gestational diabetes mellitus. Obstet Gynecol. 2013;122(2 Pt 1):406-16.

6. Setji TL, Brown AJ, Feinglos MN. Gestational Diabetes Mellitus. Clin Diabetes. 2005;23(1):17-24.

7. Nielsen KK, de Courten M, Kapur A. The urgent need for universally applicable simple screening procedures and diagnostic criteria for gestational diabetes mellitus--lessons from projects funded by the World Diabetes Foundation. Glob Health Action. 2012;5.

8. Landon MB, Gabbe SG. Gestational diabetes mellitus. Obstet Gynecol. 2011;118(6):1379-93.

9. Kjos SL, Peters RK, Xiang A, Henry OA, Montoro M, Buchanan TA. Predicting future diabetes in Latino women with gestational diabetes. Utility of early postpartum glucose tolerance testing. Diabetes. 1995;44(5):586-91.

10. Prutsky GJ, Domecq JP, Sundaresh V, Elraiyah T, Nabhan M, Prokop LJ, et al Screening for Gestational Diabetes: A Systematic Review and Meta-Analysis. J Clin Endocrinol Metab. 2013;98(11):4311-8.

11. HAPO Study Cooperative Research Group, Metzger BE, Lowe LP, Dyer AR, Trimble ER, Chaovarindr U, et al. Hyperglycemia and adverse pregnancy outcomes. N Engl J Med. 2008;358(19):1991-2002.

12. International Association of Diabetes and Pregnancy Study Groups Consensus Panel, Metzger BE, Gabbe SG, Persson B, Buchanan TA, Catalano PA, et al. International association of diabetes and pregnancy study groups recommendations on the diagnosis and classification of hyperglycemia in pregnancy. Diabetes Care. 2010;33(3):676-82.

13. Cortés H, Ocampo I, Villegas A. Prevalencia de diabetes mellitus gestacional en una población de Medellín de 1999 - 2000: valor predictivo positivo de la prueba tamiz y comparación de los criterios de la NDDG y la ADA. Rev Colomb Obstet Ginecol. 2002;53(1):81-5.

14. Campo-Campo MN, Posada-Estrada G, Betancur-Bermúdez LC, Jaramillo-Quiceno DM. Factores de riesgo para diabetes gestacional en población obstétrica en tres instituciones de Medellín, Colombia. Estudio de casos y controles. Rev Colomb Obstet Ginecol. 2012;63(2):114-8.

15. Metzger BE, Buchanan TA, Coustan DR, de Leiva A, Dunger DB, Hadden DR, et al. Summary and recommendations of the Fifth International WorkshopConference on Gestational Diabetes Mellitus. Diabetes Care. 2007;30 Suppl 2:S251-60.

16. Long $\mathrm{H}, \mathrm{Cundy} \mathrm{T}$. Establishing consensus in the diagnosis of gestational diabetes following HAPO: where do we stand? Curr Diab Rep. 2013;13(1):43-50.

17. Langer 0 , Umans JG, Miodovnik M. Perspectives on the proposed gestational diabetes mellitus diagnostic criteria. Obstet Gynecol. 2013;121(1):177-82.

18. Vandorsten JP, Dodson WC, Espeland MA, Grobman WA, Guise JM, Mercer BM, et al. NIH consensus development conference: diagnosing gestational diabetes mellitus. NIH Consens State Sci Statements. 2013;29(1):1-31.

19. Thompson D, Berger H, Feig D, Gagnon R, Kader T, Keely E, et al. Diabetes and Pregnancy. Can J Diabetes. 2013;37, Supplement 1:S168-83.

20. Crowther CA, Hiller JE, Moss JR, McPhee AJ, Jeffries WS, Robinson JS, et al. Effect of treatment of gestational diabetes mellitus on pregnancy outcomes. N Engl J Med. 2005;352(24):2477-86.

21. Landon MB, Spong CY, Thom E, Carpenter MW, Ramin SM, Casey B, et al. A multicenter, randomized trial of treatment for mild gestational diabetes. $\mathrm{N}$ Engl J Med. 2009;361(14):1339-48.
22. American Diabetes Association. 2. Classification and Diagnosis of Diabetes. Diabetes Care. 2015;38(Supplement 1):S8-16.

23. National Collaborating Centre for Women's and Children's Health. Diabetes in pregnancy. Management of diabetes and its complications from preconception to the postnatal period. [Internet]. NICE Clinical Guidelines; 2015 [cited 2015 Jun 21]. Available from: http://www.nice.org.uk/guidance/ng3

24. Alianza CINETS. Guía de práctica clínica para el diagnóstico, tratamiento y seguimiento de la Diabetes gestacional [Internet]. Guías de Practica Clínica - Ministerio de Salud; 2015 [cited 2015 Jun 21]. Available from: http://medicina.javeriana.edu.co/documents/3185897/3187458/Recomendaciones+ diabetes+gestacional.pdf/90b49f43-3f57-44a1-a147-3053e07c6470

25. Hartling L, Dryden DM, Guthrie A, Muise M, Vandermeer B, Donovan L. Benefits and harms of treating gestational diabetes mellitus: a systematic review and meta-analysis for the U.S. Preventive Services Task Force and the National Institutes of Health Office of Medical Applications of Research. Ann Intern Med. 2013;159(2):123-9.

26. Hawkins JZS, Wing D. Abnormal glucose metabolism: diagnosis and management in the ambulatory setting. Clin Obstet Gynecol. 2012;55(3):731-43.

27. Van Leeuwen M, Louwerse MD, Opmeer BC, Limpens J, Serlie MJ, Reitsma JB, et al. Glucose challenge test for detecting gestational diabetes mellitus: a systematic review. BJOG Int J Obstet Gynaecol. 2012;119(4):393-401.

28. Esakoff TF, Cheng YW, Caughey AB. Screening for gestational diabetes: different cut-offs for different ethnicities? Am J Obstet Gynecol. 2005;193(3 Pt 2):1040-4.

29. Naylor CD, Sermer M, Chen E, Farine D. Selective Screening for Gestational Diabetes Mellitus. N Engl J Med. 1997;337(22):1591-6.

30. Griffin ME, Coffey M, Johnson H, Scanlon P, Foley M, Stronge J, et al. Universal vs. risk factor-based screening for gestational diabetes mellitus: detection rates, gestation at diagnosis and outcome. Diabet Med J Br Diabet Assoc. 2000;17(1):26-32.

31. Cosson E, Benbara A, Pharisien I, Nguyen MT, Revaux A, Lormeau B, et al. Diagnostic and Prognostic Performances Over 9 Years of a Selective Screening Strategy for Gestational Diabetes Mellitus in a Cohort of 18,775 Subjects. Diabetes Care. 2013;36(3):598-603.

32. McCarthy AD, Curciarello R, Castiglione N, Tayeldín MF, Costa D, Arnol V, et al. Universal versus selective screening for the detection, control and prognosis of gestational diabetes mellitus in Argentina. Acta Diabetol. 2010;47(2):97103.

33. Avalos GE, Owens LA, Dunne F, ATLANTIC DIP Collaborators. Applying current screening tools for gestational diabetes mellitus to a European population: is it time for change? Diabetes Care. 2013;36(10):3040-4.

34. Teh WT, Teede HJ, Paul E, Harrison CL, Wallace EM, Allan C. Risk factors for gestational diabetes mellitus: implications for the application of screening guidelines. Aust N Z J Obstet Gynaecol. 2011;51(1):26-30.

35. Meltzer SJ, Snyder J, Penrod JR, Nudi M, Morin L. Gestational diabetes mellitus screening and diagnosis: a prospective randomised controlled trial comparing costs of one-step and two-step methods. BJOG Int J Obstet Gynaecol. 2010;117(4):407-15.

36. Mission JF, Ohno MS, Cheng YW, Caughey AB. Gestational diabetes screening with the new IADPSG guidelines: a cost-effectiveness analysis. Am J Obstet Gynecol. 2012;207(4):326.e1-9.

37. Duran A, Sáenz S, Torrejón MJ, Bordiú E, Del Valle L, Galindo M, et al. Introduction of IADPSG Criteria for the Screening and Diagnosis of Gestational Diabetes Mellitus Results in Improved Pregnancy Outcomes at a Lower Cost in a Large Cohort of Pregnant Women: The St. Carlos Gestational Diabetes Study. Diabetes Care. 2014;37(9):2442-50.

38. Chodick G, Elchalal U, Sella T, Heymann AD, Porath A, Kokia E, et al. The risk of overt diabetes mellitus among women with gestational diabetes: a population-based study. Diabet Med. 2010;27(7):779-85.

39. Bellamy L, Casas J-P, Hingorani AD, Williams D. Type 2 diabetes mellitus after gestational diabetes: a systematic review and meta-analysis. The Lancet. 2009;373(9677):1773-9. 